\title{
DEFINING HYDROCEPHALUS AFTER ANEURYSMAL SUBARACHNOID HAEMORRHAGE
}

\author{
Waqar $\mathrm{M}^{1,2}$, Mohamed S1, Dulhanty L1, Parry-Jones A ${ }^{2,3}$, Patel HC1,2
}

Departments of ${ }^{1}$ Neurosurgery \& ${ }^{3}$ Neurology; Salford Royal NHS Foundation Trust, Manchester, United Kingdom.

${ }^{2}$ Manchester Academic Health Sciences Centre, University of Manchester, Manchester, M13 9WL, UK

\section{SUMMARY}

- A significant proportion of aSAH patients undergo CSF diversion without evidence of objective hydrocephalus.

- Bicaudex index hydrocephalus thresholds may aid in the clinical decision making in patient selection for CSF diversion.

- WFNS grade does not itself influence the degree of ventricular enlargement. However, poor WFNS grade is an independent factor associated with CSF diversion.

\section{INTRODUCTION \& AIM}

- There is no universally accepted definition of hydrocephalus in aSAH.

- A number of patients may undergo CSF diversion without clear indication.

- CSF diversion is usually in the form of an external ventricular drain (EVD)

- EVDs are associated with a high morbidity - $20 \%$ risk of infection, up to $13-40 \%$ risk of haemorrhage and $12-60 \%$ risk of misplacement [1,2]

- It is important to therefore consider the right patient for this procedure.

- A simple imaging metric to define hydrocephalus in aSAH would be useful.

- Previous efforts have used the bicaudate index - though with numerous limitations [3].

The aim of this study was to derive and test a threshold bicaudate index value above which CSF diversion should be considered.

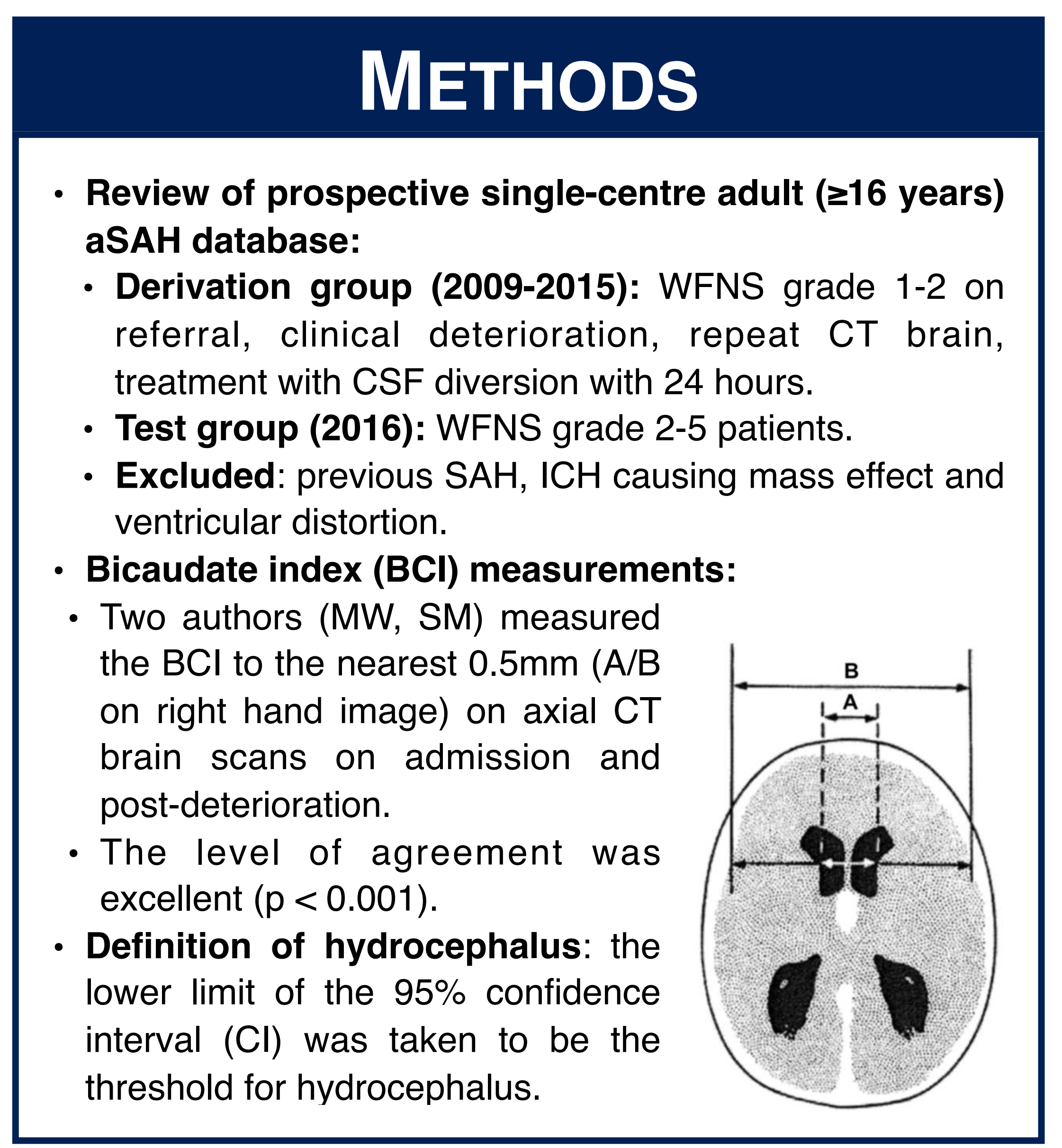

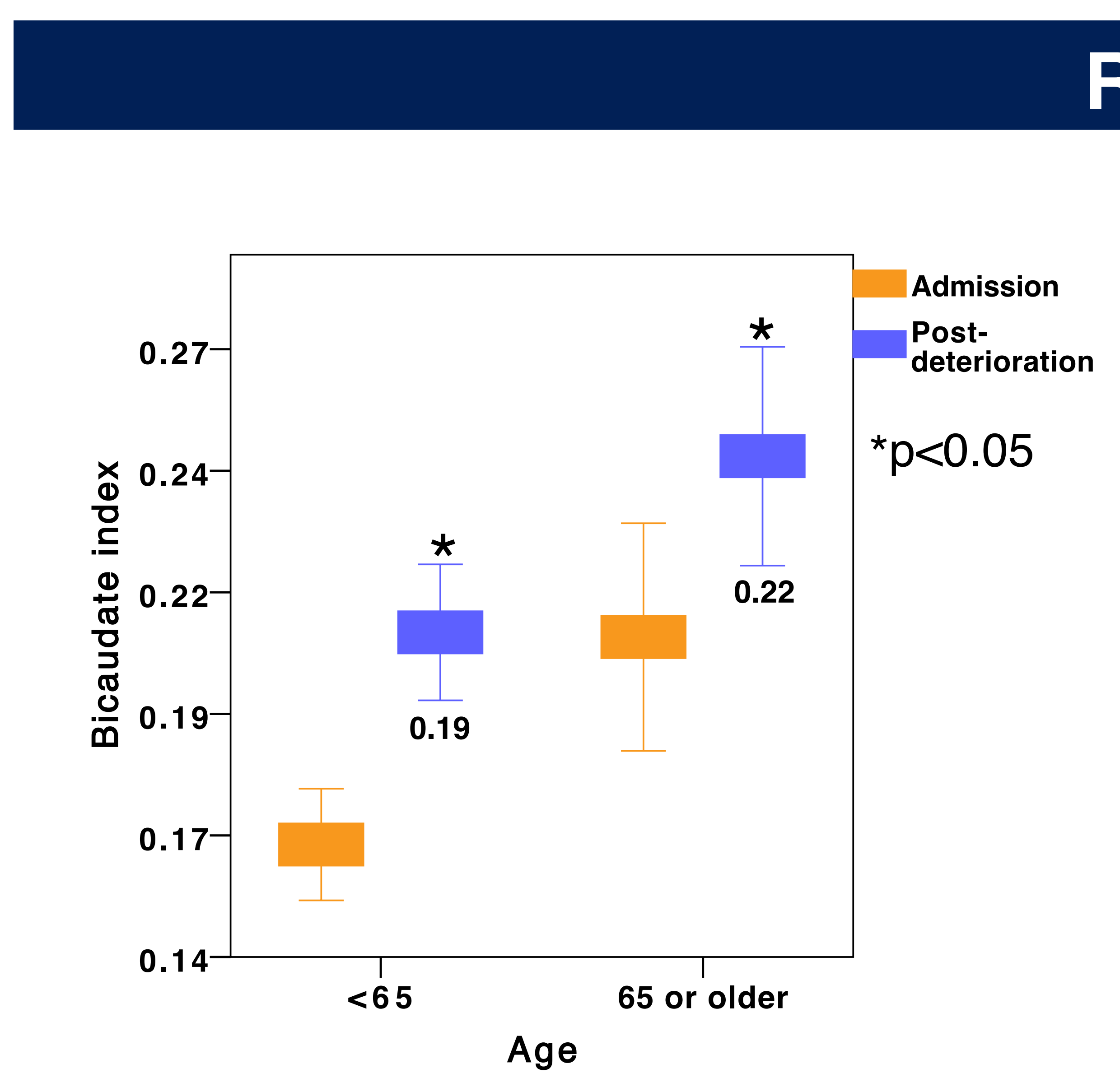

Results

Figure 1. BCl thresholds to define hydrocephalus in aSAH (derivation group). 62 patients were included with a mean age of 56 years (range 25-84 years). There was a female excess $(n=$ $42 / 62,68 \%)$. The modal WFNS grade on referral was $2(n=$ $44 / 62,71 \%$ ). The median time from admission to CSF diversion was 1 day (range 0-32 days). Post-deterioration, patients underwent an EVD $(n=57,93 \%)$ or lumbar drain $(n=5,7 \%)$. There was a significant increase in $\mathrm{BCl}$ for both age groups $(\mathrm{p}=$ $0.002, p=0.001)$. The $\mathrm{BCl}$ thresholds for hydrocephalus were taken to be the lower limits of the $95 \%$ confidence intervals for $\mathrm{BCl}$ on the post-deterioration $\mathrm{CT}$ brain scans: 0.19 ( $<65$ years) and 0.22 ( $\geq 65$ years).

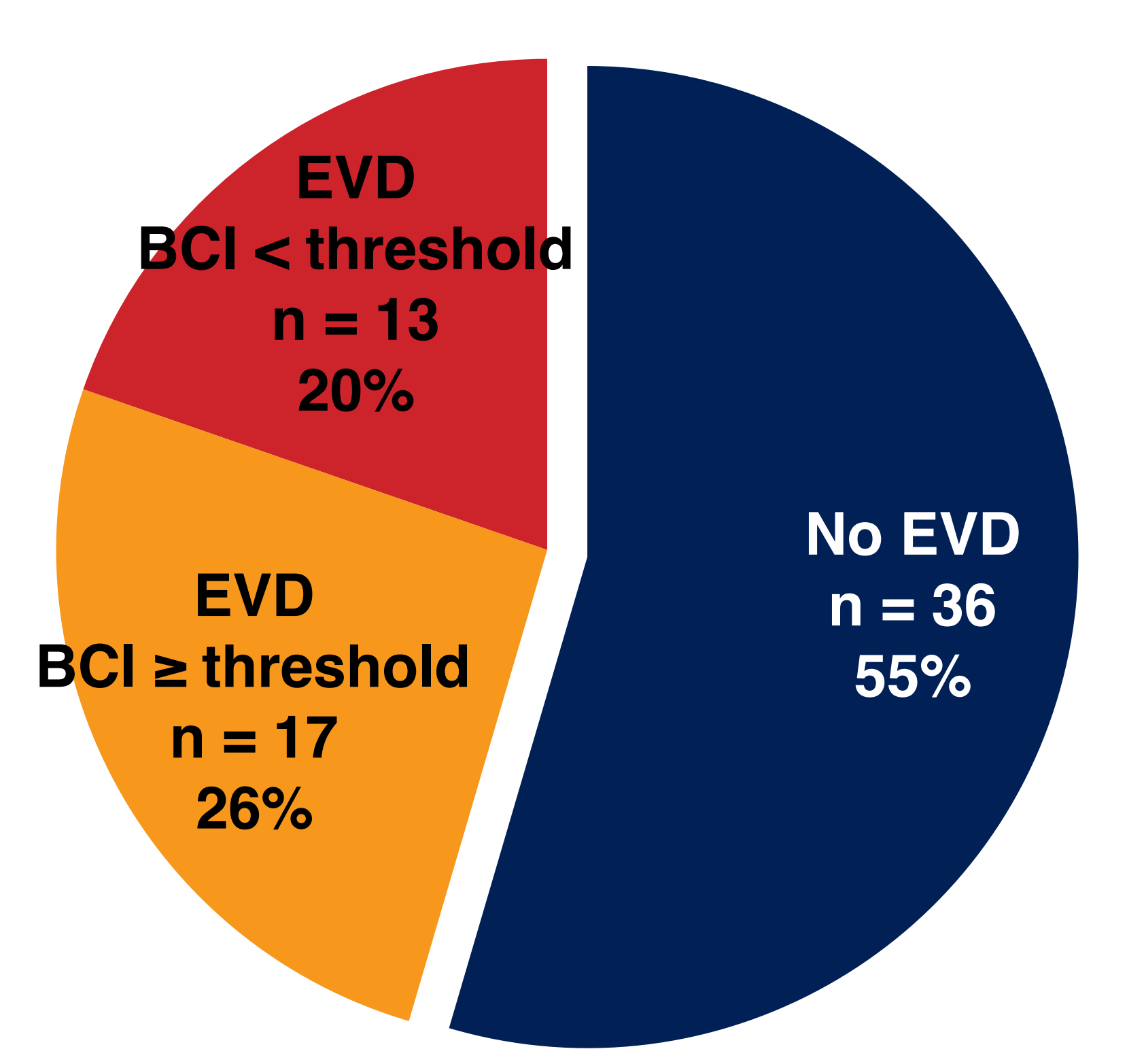

Figure 3. CSF diversion in the context of $\mathrm{BCl}$ thresholds for hydrocephalus (test group). We analysed patients in the test group and found that a substantial proportion (13/30,43\%) of CSF diversions were performed without objective evidence of hydrocephalus.

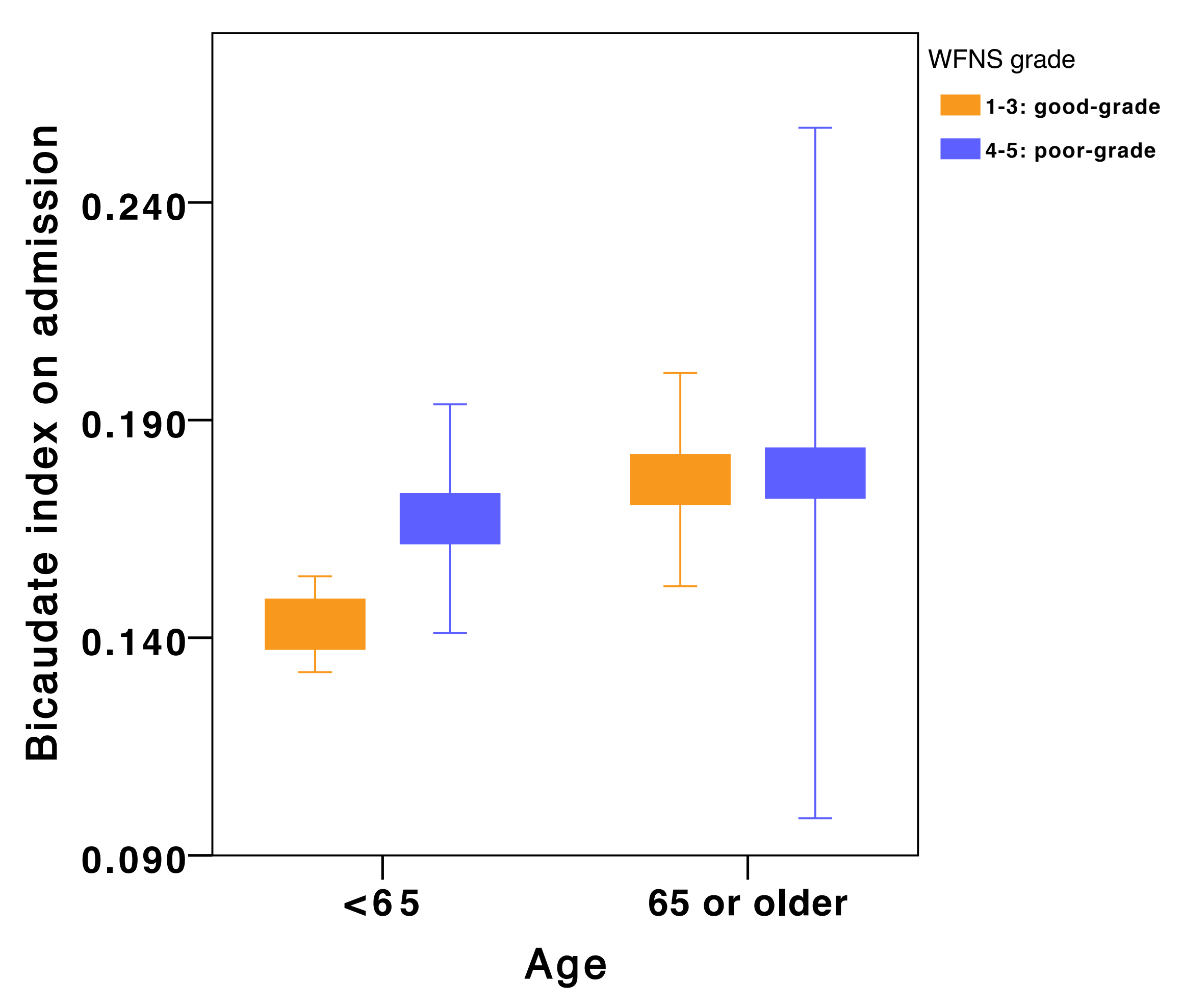

Figure 2. Differences in the $\mathrm{BCl}$ between different good and poor grade aSAH patients (test group). We compared $\mathrm{BC}$ between good and poor-grade aSAH patients in the test group. There was no significant difference in admission $\mathrm{BCl}$ between these two groups for age $<65$ years $(0.14 \pm$ SD 0.04 vs. $0.17 \pm$ SD $0.06, t=1.77, \mathrm{p}=0.09)$ and age $\geq 65$ years $(0.18 \pm \mathrm{SD} 0.06$ vs. $0.18 \pm 0.08, t=0.05, p=0.96$ )

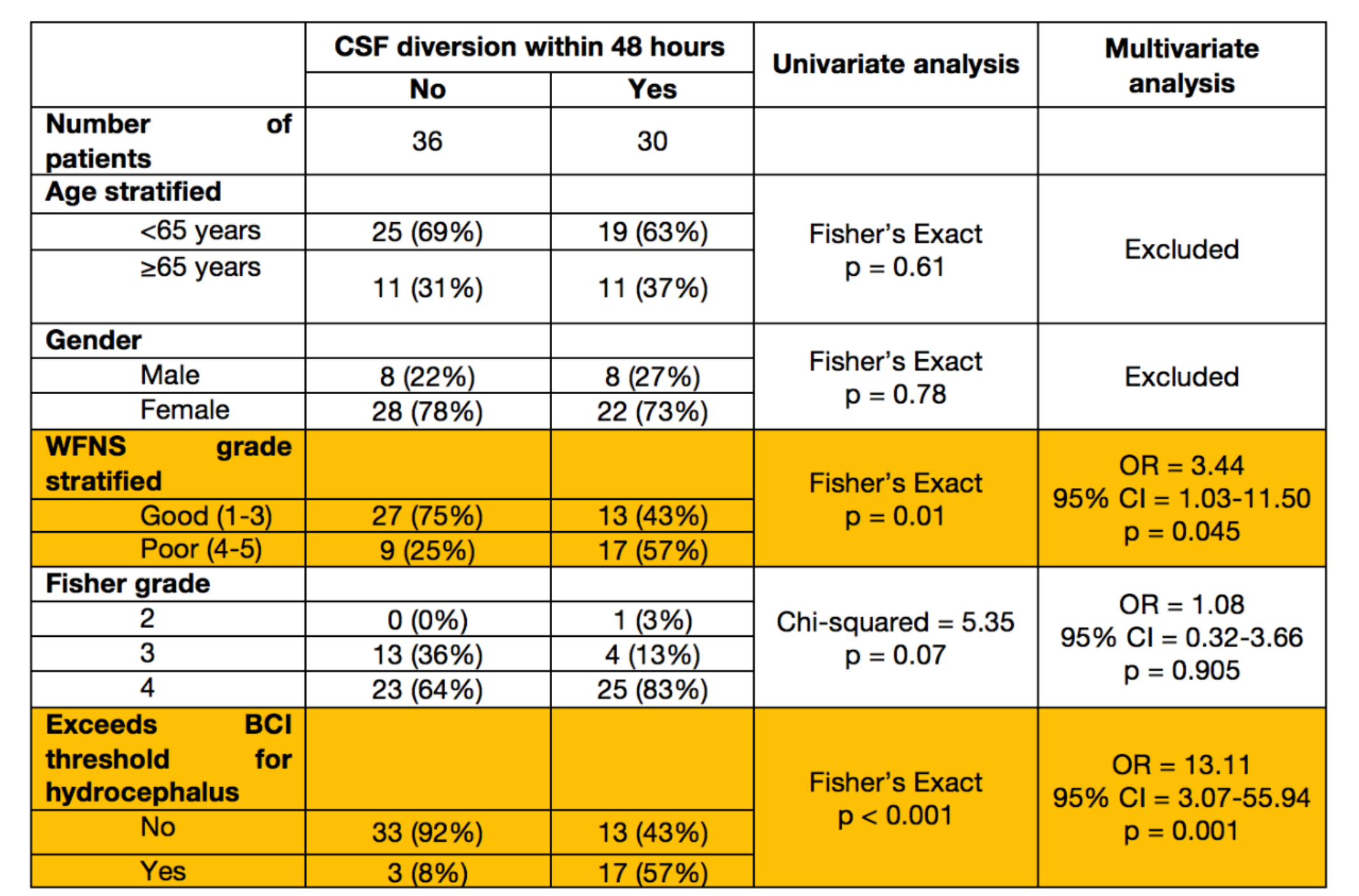

Figure 4. Factors predictive of CSF diversion in multivariate analysis (test group). Forward stepwise binary logistic regression showed that poor WFNS grade at presentation and exceeding the $\mathrm{BCl}$ threshold for hydrocephalus were predictive of CSF diversion within 48 hours. 